\title{
RESULTS OF THE IMPLEMENTATION OF JOINT MOBILIZATION TECHNIQUES TO RESTORE MOBILITY OF THE KNEE COMPLEX IN PATIENTS AFTER PARTIAL MENISCECTOMY
}

\author{
Rostislav Kostov ${ }^{1}$, Biserka Ilieva ${ }^{2}$ \\ ${ }^{1}$ Department of Physical Medicine, Rehabilitation, Occupational Therapy and Sports, \\ Medical University of Pleven \\ ${ }^{2}$ Department of Therapeutic Nursing Care, Medical University of Pleven
}

\begin{abstract}
BACKGROUND: The formation of primary and secondary motion deficit in the knee complex area is due to polyethiologic musculoskeletal dysfunctions as a consequence of multiple traumas, injuries and pre-clinical conditions.

INTRODUCTION: In this article we present the final effect of the application of complex soft tissue a manual treatment system for the recovery of joint kinematics in patients with a moderate and minimal protective period of rehabilitation after arthroscopic meniscectomy.

MATERIAL AND METHODS: The study was conducted in 2005-2012 at three medical centers in Bulgaria: Blagoevgrad, Sofia and Pleven. The study included a total of 110 patients divided into three groups (Control Group, Experimental Group I and Experimental Group II) which studied the effect of the topical application of the manual therapeutic techniques compared to the traditional rehabilitation methods applied. In order to test the efficacy of a treatment approach in the three groups of patients, the results have been processed by the method of variational analysis.

RESULTS: After an analysis of the results we found a significantly fuller and without residual short violations recovery for all controlled parameters in patients who have implemented comprehensive manual therapeutic treatment compared with the patients from the control group.

CONCLUSION: Application of adequate physiological and pedagogically grounded complex rehabilitation is required in patients after arthroscopic meniscectomy model with motor deficits in tractable routine rehabilitation. Observations allow us to offer a methodology to be implemented in the general practice rehabilitation of patients after meniscal ruptures treated by arthroscopic meniscectomy and motor deficits, who cannot benefit from routine rehabilitation.
\end{abstract}

Keywords: physiotherapy, manual therapeutic techniques, arthrokinematics of the knee complex

Address for correspondence:

Rostislav Kostov

Medical University of Pleven,

1. Sv. Kliment Ohridski Str.

Pleven, Bulgaria

e-mail:rostislav_kostov@abv.bg

Received: November 2, 2015

Accepted: February 1, 2016

\section{INTRODUCTION}

One of the most common inner joint traumas in clinical practice, is that of the knee menisci $(1,2)$. The great social importance of meniscus ruptures $(3,4)$ motivates us to study the disruption in the joint mechanics and use some modern methods of musculoskeletal physiotherapy, targeting the correction of the motor deficit and the restoration of joint mechanics. 
This is the basis for building and realization of the full functional recovery in patients after arthroscopic meniscectomy in which typically applied rehabilitation does not give the desired results. We applied biomechanical and pathophysiological soft tissue methods grouped in an appropriate system, never before administered to patients with motion deficiency and arthroscopic meniscectomy.

\section{METHODOLOGY OF SCIENTIFIC RESEARCH}

\section{Purpose of the Scientific Research}

The purpose of this scientific research is a complex evaluation of the administered motion program, including manual therapeutic techniques aimed at restoring the arthrokinematics of the knee in patients with motion deficiency and arthroscopic meniscectomy, as well as its clinical approbation.

\section{Tasks of the Study}

* Analysis of the pathomechanical factors for motion deficiency of the knee in patients with meniscus ruptures, after arthroscopic meniscectomy surgery.

* Study and analysis of the mechanisms for disrupting the arthrokinematics of the knee in patients after arthroscopic meniscectomy.

* Analysis and correction of the musculoskeletal dysfunctions violating knee arthrokinematics after arthroscopic meniscectomy surgery.

* Developing a kinesiology- and physiologybased approach for recovery of knee kinematics after arthroscopic meniscectomy, in cases of motion dysfunction unaffected by routine rehabilitation.

* Applying the combined method on suitable patients grouped by identical criteria.

* Analysis of the results and conclusions regarding the effect of the motion program for recovery of knee kinematics after arthroscopic meniscectomy and motion deficiency.

\section{METHODS OF MONITORING}

The following methods have been used to register the signs of the observation.

\section{Clinical Observation}

It covers the history of the condition including description of the mechanism of the injury, clinical diagnosis and concomitant pathologies (if any), the date and type of surgical treatment.

\section{Functional Diagnostic Methods}

With the aim of establishing the rehabilitation potential, we selected monitoring the effects of the treatment (angulometry, girth measurements, manual muscle testing, static muscular endurance tests, gait testing) as well as determining the contraindications for applying the method.

\section{MATHEMATICAL AND STATISTI- CAL METHODS}

In order to the efficiency of the therapeutic approach in the three patient groups, we processed the results using the variational analysis method. The software packages used for the analysis are: MS OFFICE 2010 applications Excel 2010 and SPSS 17.0. For this purpose we entered the data of each patient of the three groups reflecting only the beginning and end results. We determined the guaranteed probability $(\mathrm{P})$ for each of the survey indicators and the level of significance ( $\alpha$ ) reflecting the risk of errors when accepting a true alternative hypothesis.

The general aggregate of sampling is considered by the variation analysis, therefore the decisions have got a probabilistic nature. Therefore, in our study we determined the guarantee probability $(\mathrm{P})$ for each of the indicators and the level of significance $(\alpha)$ reflects the risk of errors in the acceptance of a true alternative hypothesis. We used the standard values for the guarantee probability $(\mathrm{P})$ and a significance level $(\alpha)$ :

* $P=95 \%$, which corresponds to $\alpha=0.05$ (5\% error margin).

* $\mathrm{P}=99 \%$, which corresponds to $\alpha=0.01$ ( $1 \%$ error margin).

* $\mathrm{P}=99.9 \%$, which corresponds to $\alpha=0.001(0.1 \%$ error margin).

For statistical verification of results we have used the independent Student's t-criterion calculated via a formula. To determine the tabulated value of a criterion ( criterion $\alpha$ ) it is taken from the statistical tables depending on the degrees of freedom $(\mathrm{k})$ and the level of significance $(\alpha)$. For independent samples, we calculated the degree of freedom by the standard model $(\mathrm{k}=\mathrm{n} 1+\mathrm{n} 2-2$, where $\mathrm{n}=$ the number of compared cases). To establish the level of significance ( $\alpha$ ) we compared the calculated t-criteria at 
Results of the implementation of the joint mobilization techniques to restore mobility of the knee complex in patients after partial ...

standard tabular value. In order to take decisions, we compared the empirical value (calculated according to data from the sample) with the tabulated value of the criterion.

\section{Number of Observed Patients, Time and Place of the Study}

The total number of patients under observation was 110 people. They were selected randomly according to the indications for the experimental method. They were divided into three groups:

1. Experimental Group I. It consists of 30 people treated by the methods of classical physiotherapy used after arthroscopic meniscectomy $(1,5)$ surgery including a pain -relieving mobilization stretching of the knee.

2. Experimental Group II. It includes 50 patients treated by the methods of classical physiotherapy used after arthroscopic meniscectomy surgery including a pain- relieving mobilization stretching, Mulligan (6) and Maitland (7) joint mobilization techniques and mobilizing muscular stretching of the knee. A controlled group is used to compare the results of the analysis.

3. Control Group. It includes 30 patients mainly treated with active medical means in accordance with the concept of classical physiotherapy procedures $(1,5)$ during the moderately protective period.

\section{COMPARATIVE ANALYSIS AND DISCUSSION OF CHANGES IN THE FUNCTIONAL AND DIAGNOSTIC INDICATORS IN THE THREE PA- TIENT GROUPS}

To determine the final effect of applying the tested softtissue manual therapeutic method to restore the arthrokinematics of the knee in patients with arthroscopic meniscectomy and motion deficiency. The results are processed and analyzed according to the standard principles for conducting such studies.

\section{Results of Goniometry of the Knee}

To summarize the results of the study of knee flexion: the greatest improvement is in patients from EG II $\left(38.60^{\circ}\right)$, followed by EG I $\left(27.33^{\circ}\right)$ and CG $\left(21.83^{\circ}\right)$. When comparing the results of CG and EG I there is a distinct improvement in patients from EG I with $5.5^{\circ}$ ( $\mathrm{t}=5.38$; $\mathrm{a}<0.001 ; \mathrm{P}=99.9 \%$ ). When comparing the results of CG and EG II there is a difference of $16.77^{\circ}(\mathrm{t}=17.65 ; \mathrm{a}<0.001 ; \mathrm{P}=99.9 \%)$ in favor of the tested complex method. When comparing the results of EG I and EG II there is a distinct improvement in patients from EG II of $11.27^{\circ}(\mathrm{t}=15.72$; $\mathrm{a}<0.001$; $\mathrm{P}=99.9 \%$ ) (Fig. 1, Table 1).

From the study of knee extension in patients from EG I there is an average improvement of $13.83^{\circ}$

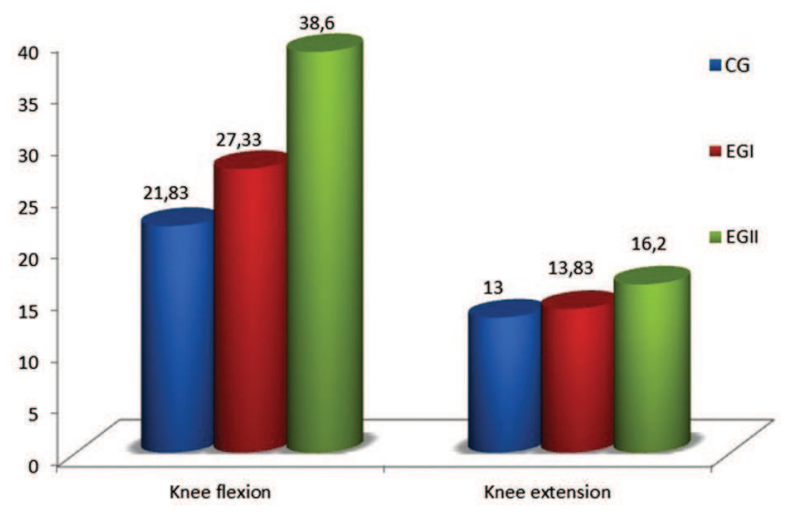

Fig. 1. Average improvement of angular movements in the sagittal plane $(\Delta X)$ in three groups of patients. CG - control group; EG I - experimental group I; EG II Experimental Group II

Table 1. Statistical analysis of the results of anglometriy on knee complex at patients in all three groups. CG - control group; EG I - Experimental group II; EG II - Experimental group II.

\begin{tabular}{|c|c|c|c|c|c|c|}
\hline Movement & Group & $\Delta \mathbf{X}$ & S & t & a & P\% \\
\hline \multirow{3}{*}{ Flexion } & CG & 21.83 & 4.64 & 25.77 & $<0.001$ & $99.9 \%$ \\
\hline & EG I & 27.33 & 3.14 & 47.61 & $<0.001$ & $99.9 \%$ \\
\hline & EG II & 38.60 & 3.03 & 89.86 & $<0.001$ & $99.9 \%$ \\
\hline \multirow{3}{*}{ Extension } & CG & 13 & 4.27 & 16.65 & $<0.001$ & $99.9 \%$ \\
\hline & EG I & 13.83 & 3.63 & 20.81 & $<0.001$ & $99.9 \%$ \\
\hline & EG II & 16.20 & 2.77 & 41.24 & $<0.001$ & $99.9 \%$ \\
\hline
\end{tabular}


$(\mathrm{S}=3.63)$. The results have a high degree of statistical authenticity here as well $(\mathrm{t}=20.81 ; \mathrm{a}<0.001 ; \mathrm{P}=99.9 \%)$ (fig.1, table 1). The average improvement of extension in EG II is $16.20^{\circ}(\mathrm{S}=2.77)$. The statistical authenticity is very high ( $\mathrm{t}=41.24 ; \mathrm{a}<0.001 ; \mathrm{P}=99.9 \%)$ (fig.1, table 1). In patients from CG there is an average improvement of $13^{\circ}(\mathrm{S}=4.27)$. The statistical authenticity is very high ( $\mathrm{t}=16.65 ; \mathrm{a}<0.001 ; \mathrm{P}=99.9 \%$ ) (Fig. 1, Table 1).

To summarize the results of the study of knee extension: the greatest improvement is in patients from EG II $\left(16.20^{\circ}\right)$, followed by EG I $\left(13.83^{\circ}\right)$ in comparison to CG $\left(13^{\circ}\right)$. The analysis of the results shows a significant difference between the results of improvement of knee extension in CG and EG I $\left(\Delta \mathrm{X}=0.83^{\circ} ; \mathrm{t}=0.81 ; \alpha>0.05 ; \mathrm{P}<95 \%\right)$. The result of the measured indicator between EG II in comparison to the other two groups has a statistically significant difference $(\mathrm{a}<0.001 ; \mathrm{P}=99.9 \%)$. The average difference of improvement of knee extension in patients from EG I and EG II is $2.37^{\circ}(\mathrm{t}=3.07 ; \alpha<0.001$; $\mathrm{P}=99.9 \%$ ) (Fig.1, Table 1); in comparison to the results of CG where the average difference of $3.2^{\circ}$ in favor of the results of patients from EG II $(\mathrm{t}=3.66$; $\alpha<0.001 ; \mathrm{P}=99.9 \%$ ) (Fig. 1, Table 1).

After the compared analysis of the results from the standard goniometry in the three patient groups
2. The conclusion from the analysis, regarding the effectiveness of the tested manual therapeutic method, is that applying elements from the joint mobilization techniques of Mulligan and Maitland, pain relieving and analytical muscle stretching is the most effective combination to restore the physiological motion of the knee in patients with arthroscopic meniscectomy and motion deficiency.

\section{Hip girth measurement}

When we measure hip circumference of standard $1^{\text {st }}$ level (5-7 cm cranial from tuberositas tibiae) it occurs as a distinct outcome in patients - contingent EG II $(1.42 \mathrm{~cm})$, followed by those in the composition of the EG I $(1.18 \mathrm{~cm})$ compared with CG $(1.12 \mathrm{~cm})$. When we measure hip circumference of the standard $2^{\text {nd }}$ level (10-12 cranial from tuberositas tibiae), we establish the greatest improvement in patients from EG II $(2.48 \mathrm{~cm})$, followed by the results of EG I $(2.03 \mathrm{~cm})$ and CG $(1.95 \mathrm{~cm})$. Results of the study are presented in Table 2.

\section{Manual muscle testing}

To verify the degree of muscle weakness in the patients - contingent of study, we tested the major muscles in the knee complex: mm. quadriceps femoris; tensor fascia latae; semimembranosus; semi-

Table 2. Statistical analysis of the results obtained by measuring the hip laps at patients in all three groups.

\begin{tabular}{lcc|c|c|cc} 
Level Measurement & Group & $\mathbf{\Delta X}$ & $\mathbf{S}$ & $\mathbf{t}$ & $\alpha$ & $\mathbf{P} \%$ \\
\multirow{3}{*}{$1^{\text {st }}$ level } & CG & 1.12 & 0.22 & 28.43 & $<0.001$ & $99.9 \%$ \\
& EG I & 1.18 & 1.18 & 26.44 & $<0.001$ & $99.9 \%$ \\
& EG II & 1.42 & 0.19 & 54.22 & $<0.001$ & $99.9 \%$ \\
\multirow{2}{*}{$2^{\text {nd }}$ level } & CG & 1.95 & 0.30 & 35.16 & $<0.001$ & $99.9 \%$ \\
& EG I & 2.03 & 0.13 & 87.79 & $<0.001$ & $99.9 \%$ \\
& EG II & 2.48 & 0.20 & 87.23 & $<0.001$ & $99.9 \%$ \\
\hline
\end{tabular}

we made the following summaries and discussion:

1. All patients taking part in this study have been found to have a significant positive dynamic in the restoration of the knee motion. The analysis of the results shows a significant improvement in patients from Experimental Group II, followed by Experimental Group I and the Control Group. tendinosus and biceps femoris. We present the data in a tabular form (Table. 3).

\section{Testing of isometric muscle endurance}

In order to verify the final effect from the treatment, respectively, the restored contractile muscle performance in isometric mode, we have applied the analytical test of timing of individual sections for $\mathrm{m}$. quadriceps femoris. The presented results are synthesized in Table 4. 
Results of the implementation of the joint mobilization techniques to restore mobility of the knee complex in patients after partial ...

Table. 3. Statistical analysis of the results from MMT of major muscles in the area of the knee, in patients from all three groups

\begin{tabular}{|c|c|c|c|c|c|}
\hline Group & Tested muscle & $\Delta \mathbf{X}$ & $\mathbf{S}$ & $\mathbf{t}$ & $\mathrm{P} \%$ \\
\hline \multirow{4}{*}{ CG } & M. quadriceps femoris & 0.60 & 0.20 & 16.15 & 99.9 \\
\hline & M. tensor fascia latae & 0.70 & 0.25 & 15.38 & 99.9 \\
\hline & MM. semitendinosus et semimembranosus & 0.78 & 0.25 & 17.02 & 99.9 \\
\hline & M. biceps femoris & 0.75 & 0.25 & 16.15 & 99.9 \\
\hline \multirow{4}{*}{ EG I } & M. quadriceps femoris & 0.75 & 0.28 & 14.35 & 99.9 \\
\hline & M. tensor fascia latae & 0.81 & 0.20 & 16.08 & 99.9 \\
\hline & MM. semitendinosus et semimembranosus & 0.88 & 0.28 & 17.02 & 99.9 \\
\hline & M. biceps femoris & 0.86 & 0.29 & 16.26 & 99.9 \\
\hline \multirow{4}{*}{ EG II } & M. quadriceps femoris & 1.07 & 0.17 & 43.17 & 99.9 \\
\hline & M. tensor fascia latae & 1.07 & 0.18 & 43.17 & 99.9 \\
\hline & MM. semitendinosus et semimembranosus & 1.09 & 0.19 & 39.72 & 99.9 \\
\hline & M. biceps femoris & 1.08 & 0.18 & 41.24 & 99.9 \\
\hline
\end{tabular}

Table. 4. Statistical analysis of the results obtained after testing the isometric muscular strength in the patients of the three groups

\begin{tabular}{|c|c|c|c|c|c|}
\hline Group & Tested muscle & $\Delta \mathrm{X}$ & $\mathbf{S}$ & $\mathbf{t}$ & $\mathrm{P} \%$ \\
\hline \multirow{3}{*}{ CG } & M. rectus femoris & 25.33 & 4.90 & 28.31 & 99.9 \\
\hline & M. vastus medialis & 28.83 & 3.39 & 46.52 & 99.9 \\
\hline & M. vastus lateralis & 24.66 & 3.92 & 34.42 & 99.9 \\
\hline \multirow{3}{*}{ EG I } & M. rectus femoris & 29.03 & 6.40 & 24.81 & 99.9 \\
\hline & M. vastus medialis & 33.4 & 2.15 & 84.72 & 99.9 \\
\hline & M. vastus lateralis & 31.8 & 1.76 & 98.43 & 99.9 \\
\hline \multirow{3}{*}{ EG II } & M. rectus femoris & 37.04 & 2.05 & 127.14 & 99.9 \\
\hline & M. vastus medialis & 39.58 & 1.40 & 199.76 & 99.9 \\
\hline & M. vastus lateralis & 38.88 & 1.76 & 155.44 & 99.9 \\
\hline
\end{tabular}

Table 5. Statistical analysis of the results of testing of locomotor abilities to overcome the distance of $5 \mathrm{~m}$, without facilities in patients in all three groups

\begin{tabular}{l|c|c|ccc}
\hline Indicator & Group & $\boldsymbol{\Delta X}$ & $\mathbf{S}$ & $\mathbf{t}$ & $\mathrm{P} \%$ \\
\hline \multirow{4}{*}{ Step width } & EG & 10.86 & 1.25 & 47.53 & 99.9 \\
& EG I & 12.3 & 1.11 & 60.21 & 99.9 \\
& CG & 2.5 & 0.50 & 26.92 & 99.9 \\
Number & EG I & 2.36 & 0.49 & 26.44 & 99.9 \\
of steps & EG II & 3.12 & 0.32 & 67.20 & 99.9 \\
\hline \hline
\end{tabular}

Testing gait without facilities

1. Testing the step length of the operated lower limb to cover the distance of $5 \mathbf{~ m}$

Analysis of results showed the clear positive dynamics in patients EG II (19.26 cm.), followed by EG I $(12.3 \mathrm{~cm}$.) and CG $(10.86 \mathrm{~cm}$.).

\section{Testing the number of steps to cover the dis-} tance from $5 \mathrm{~m}$

Analysis of results showed the greatest improvement of controlled parameters in patients from EG II (3.12 n.) followed by CG (2.5 n.) and CG I (2.3 n.). The data are synthesized in Table 5. 


\section{CONCLUSIONS}

Based on the results of the study, we present the following conclusions:

1. Application of adequate physiological and pedagogically grounded physiotherapy (PT) is required in patients after arthroscopic meniscectomy model with motor deficits who show no improvement with routine rehabilitation.

2. Each of the three PT complex speeds up recovery and return patients to everyday life.

3. The inclusion of a proximal mobilization stretching (in addition to traditional physical therapy) accelerates functional recovery of the knee complex (by analytical impact on the truncated structures).

4. The most effective is a complex manual-therapeutic approach, combined with appropriate stretching techniques and ability to perform in terms of proximal or distal fixation, for a correction of the motor deficit.

5. Our structured and approved methodology is not only convenient and easy to use but highly effective in patients after arthroscopic meniscectomy and shaped motor dysfunction. It reduces arthrogenic pain, increases the volume of movement, restores intra-articular and physiological mobility, stimulates muscle trophicity, improves isometric muscular endurance, increases the muscle strength and overcomes muscle weakness, produces arthrokinematic recovery of the knee complex, and improves the stability gait.

Observations allow us to offer a methodology to be implemented in the general practice rehabilitation of patients after meniscal ruptures treated by arthroscopic meniscectomy and motor deficits who cannot benefit from routine rehabilitation.

\section{REFERENCES}

1. Urquhart MW, JA. O'Leary, JR. Giffin. Meniscal injuries: 1. Meniscal injuries in the adult.In DeLee J (ed.), DeLee and Drez's Orthopaedic Sports Medicine: Principles and Practice.-Philadelphia: Saunders. 2: 87-96. 2003.

2. Lee SJ, Aadalen KJ, Malaviya P, Lorenz EP, Hayden JK, Farr J, Kang RW, Cole BJ. Tibiofemoral contact mechanics after serial medial meniscectomies in the human cadaveric knee. Am J Sports Med. 2006, 34:1334-1344.

3. McLaughin, J, et al. Rehabilitation after meniscus repair. J. Orthopedics. 1994, 17:463.

4. Seto, JL, Brewster, CE. Rehabilitation of meniscal injuries. In Greenfield, BH (ed) Rehabilitation of the Knee: A Problem-Solving Approach.- Philadelphia: FA Davis.1993, pp. 381-409.

5. Boyce, DA, Hanley, ST: Functional based rehabilitation of the knee after partial meniscectomy or meniscal repair. Orthop Phys Ther Clin North Am. 1994, Vol. 3. p. 555.

6. Mulligan, BR: Manual Therapy "NAGS," "SNAGS," "MWM's" etc, ed 4. Plane View Press, Wellington, 1999.

7. Maitland G, E. Hengeveld, K. Banks. Maitlands. Peripheral Manipulation.- Elsevier: Butterworth, 2005. 